Article

\title{
Interface-Active Metal Organic Frameworks for Knoevenagel Condensations in Water
}

\author{
Yanmei Zhang ${ }^{1}{ }^{(\mathbb{D}}$, Xiang Zhang ${ }^{1}$, Rixia Bai ${ }^{1, *}$, Xiyan $\mathrm{Hou}^{1}$ and Jun $\mathrm{Li}^{2, *}$ \\ 1 Life Science College, Dalian Nationalities (Minzu) University, Economical and Technological Development \\ Zone, Dalian 116600, China; ymzhang@dlnu.edu.cn (Y.Z.); zhangxiang8703@126.com (X.Z.); \\ xyhous@dlnu.edu.cn (X.H.) \\ 2 State Key Laboratory of Catalysis, Dalian Institute of Chemical Physics, Chinese Academy of Science, \\ P.O. Box 110, Dalian 116023, China \\ * Correspondence: brx@dlnu.edu.cn (R.B.); jli@dicp.ac.cn (J.L.); Tel.: +86-411-87532759 (R.B.); \\ +86-411-84379302 (J.L.); Fax: +86-411-87618179 (R.B. \& J.L.)
}

Received: 5 July 2018; Accepted: 27 July 2018; Published: 1 August 2018

check for updates

\begin{abstract}
It is desirable but challenging to locate solid catalysts at the oil-water interface to stabilize "Pickering emulsions", which is one of the promising ways to develop efficient green chemical processes. Herein, water-stable metal organic framework ZIF-8 without any chemical modification was demonstrated to be an interface-active catalyst for Knoevenagel condensation in a biphasic system. Pickering emulsion formed under the reaction conditions due to its amphiphilic property, which was beneficial to the mass transfer and led to high catalytic performance. Moreover, it can be repeatedly applied for Knoevenagel condensation for at least six successive cycles without losing its catalytic activity and framework integrity.
\end{abstract}

Keywords: metal organic framework; ZIF-8; Knoevenagel condensations; Pickering emulsion; recycle

\section{Introduction}

Using water as a solvent is one of the main ways toward the development of environmentally-friendly chemical processes. However, the low mass transfer efficiencies, ascribed to a modest interfacial contact due to the inhomogeneous mixing even under vigorous stirring, leads to poor catalytic activities in biphasic reaction systems. Although adding surfactants is a frequently-used way to increase the liquid-liquid interfacial area, it results in a negative environmental impact. Alternatively, using an interfacial solid catalyst to stabilize a "Pickering emulsion" is an emerging strategy to develop greener chemical processes. "Pickering emulsion" is an emulsion that is stabilized by interfacial particles. The catalytic efficiencies in biphasic catalytic systems could be improved greatly by the location of interfacial catalysts at the interfaces. Likewise, it could not only decrease the phase transfer limitations, but also could catalyze reactions at the interface of two immiscible solvents. Furthermore, the interfacial solid catalysts can be easily recovered from the Pickering emulsions after the reaction [1]. In line with the aforementioned properties of Pickering emulsions, a couple of interface-active particles, such as silicas [2-8], zeolites [9], carbon [10] and metal organic frameworks [11,12], were attempted to be used to stabilize Pickering emulsions.

Metal organic frameworks (MOFs) are a new class of highly-ordered porous coordination polymers that can be assembled from plenty of metal ions/clusters and multi-topic organic struts. MOFs are more attractive materials than conventional porous materials in heterogeneous catalysis, due to their exceptional modular properties, imparting ultra high porosity, structural diversity, tunable surface properties and diverse functionalities $[13,14]$. Despite progressive advances made so far in the synthesis and catalytic applications of MOFs, the instability in water has considerably limited 
these MOFs' applications in Pickering emulsions [15,16]. ZIF-8 is one of the representative examples of water-stable MOFs, which could be constructed by $\mathrm{Zn}^{2+}$ and 2-methylimidazole in organic or aqueous solvents. The efficiency of ZIF-8 has been demonstrated for heterogeneous Knoevenagel condensations [17,18], cycloadditions [19] and Friedel-Crafts acylation [20] in organic solvents. However, its catalytic efficiency in aqueous medium has not yet been investigated. Herein, as shown in Figure 1, ZIF-8 was prepared with a narrow size distribution and investigated as an interfacial catalyst to promote the Knoevenagel condensation. It is demonstrated that ZIF-8 shows a good self-assembly ability at the oil-water interface and allows one to formulate an oil-in-water $(\mathrm{O} / \mathrm{W})$ Pickering emulsion. Moreover, the interface-active catalyst can be recovered by centrifugation and reused for at least six times without obvious loss of its catalytic performance and framework integrity.

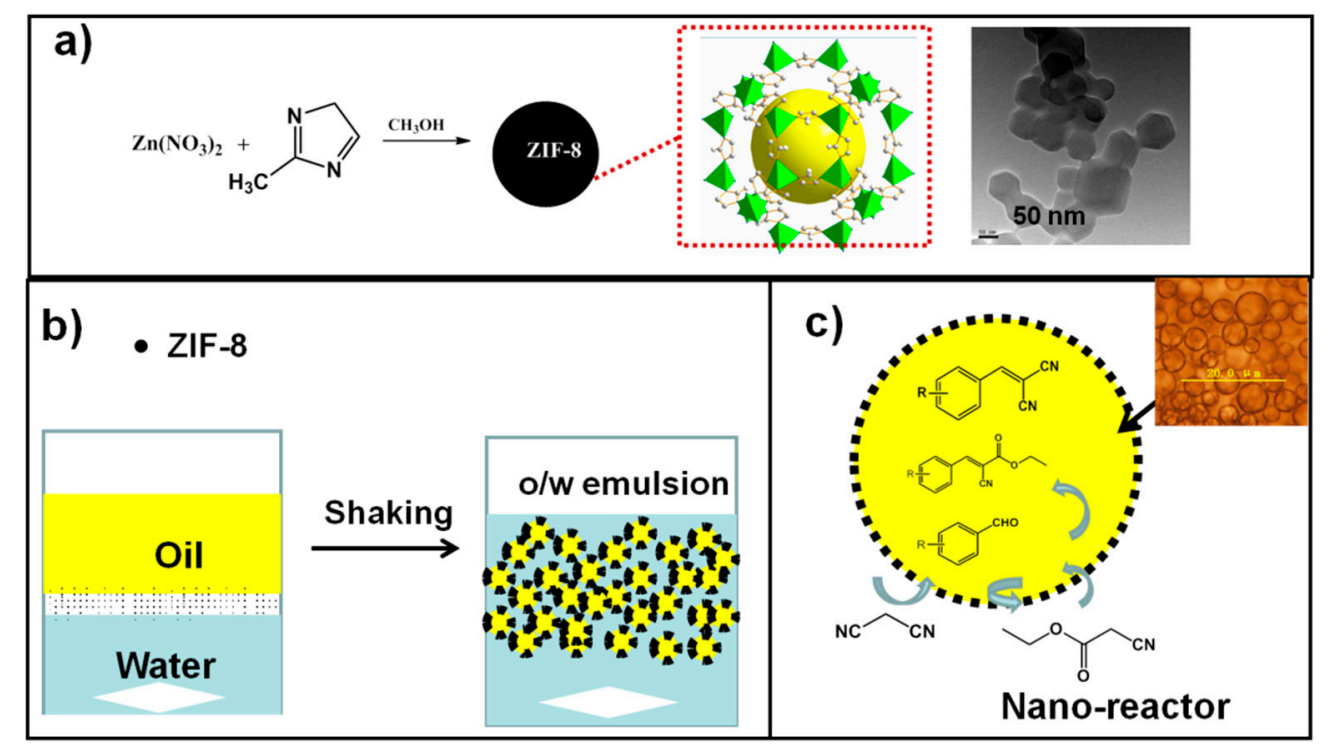

Figure 1. Schematic presentation of the synthesis of ZIF-8 nanoparticles and catalytic reactions in the Pickering emulsion. (a) Synthesis and TEM image of ZIF-8 nanoparticles; (b) Formation of Pickering emulsion stabilized by ZIF-8; (c) Schematic presentation of the Knoevenagel condensation in Pickering emulsion.

\section{Results and Discussion}

ZIF-8 nanoparticles with narrow size distributions were prepared accordingly. Scanning electron microscopy (SEM) was used to characterize their morphology. Figure 2A shows representative SEM micrographs of ZIF-8. Regular nanoparticles with a narrow size distribution (about $100 \mathrm{~nm}$ ) were observed. X-ray powder diffraction (XRD) was further used to determine the crystal structure of ZIF-8. As shown in Figure 3a, the diffraction pattern for the prepared solid has several sharp and intense peaks that can be well indexed to ZIF-8 [21]. Its structural stability in water was tested, and the diffraction pattern for recovered ZIF-8 is shown in Figure 3b. No obvious change was observed before and after treatment, indicating that it was water stable, which is a prerequisite for potential applications in an aqueous medium. Meanwhile, images of its transmission electron microscopies (TEM) further confirmed its water stability. 

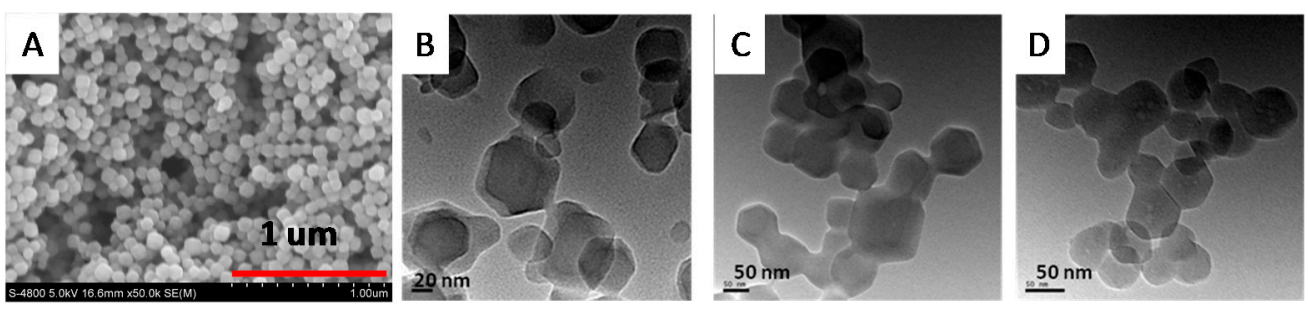

Figure 2. SEM images of fresh ZIF-8 (A) and TEM images of fresh ZIF-8 (B,C) and recovered ZIF-8 (D).

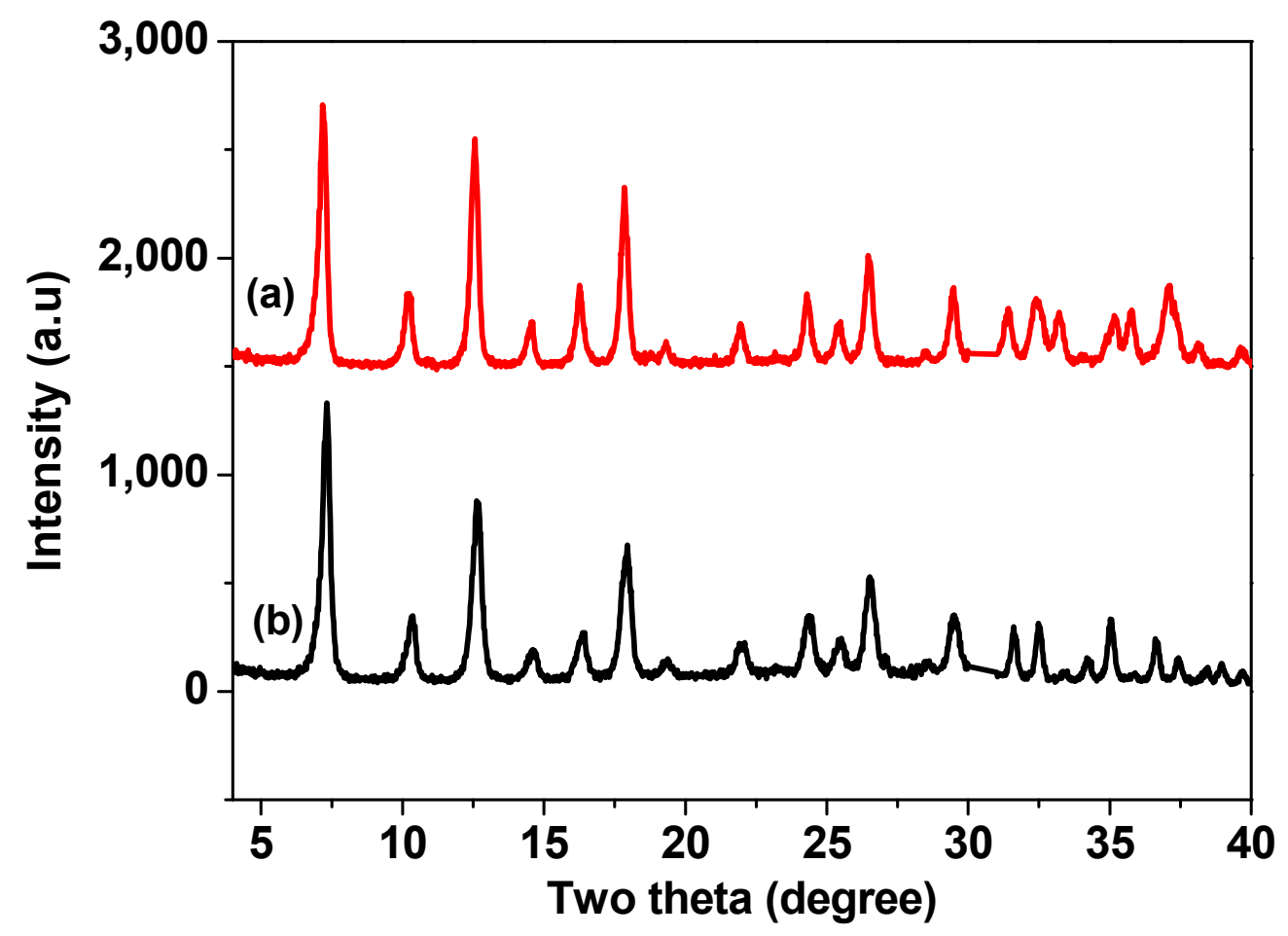

Figure 3. XRD patterns of fresh ZIF-8 (a) and recovered ZIF-8 after treatment in water (b).

To the best of our knowledge, MOFs including ZIF-8 have been confirmed to be good catalysts for the Knoevenagel condensations in organic solvents [17,18]. The stability in aqueous medium of ZIF-8 has been proven by the XRD patterns and TEM images as shown in Figures 2 and 3 in our experiments. Therefore, the activities of ZIF-8 nanoparticles for the Knoevenagel condensation between benzaldehyde and ethyl cyanoacetate in various solvents were firstly investigated, and the results are summarized in Table 1. Similar to the results observed in previous reports, ZIF-8 gave high or medium yields in polar solvents and poor results in nonpolar solvents. As we know, catalytic reaction in water or biphasic systems is a greener route than in organic solvent. To our delight, ZIF-8 could also promote the reaction in water in an efficient way. Over an $80 \%$ yield was obtained in water after $30 \mathrm{~min}$, a little bit lower than that in DMF, but higher than that in THF. Then, a water/ZIF-8/ethyl acetate system was used to examine the interfacial property of ZIF-8. After adding $2 \mathrm{~mL}$ of pure or 2-nitrobenzaldehyde dissolved ethyl acetate to $3 \mathrm{~mL}$ of water containing $0.8 \mathrm{wt} \%$ of ZIF-8 (with respect to water), as shown in Figure 4A,B, ZIF-8 is predominantly distributed at the interface between the oil and aqueous phase. After subsequent vigorous stirring or shaking, the appearances of its mixture (Figure $4 \mathrm{C}$ ) and its optical micrograph demonstrated the formation of Pickering emulsion. Optical micrographs revealed that it was an oil-in-water Pickering emulsion, and the droplets sizes were about 2-10 $\mu \mathrm{m}$. 
Table 1. Knoevenagel condensation between benzaldehyde and ethyl cyanoacetate in various solvents in the presence of ZIF-8.

\begin{tabular}{|c|c|c|c|c|c|c|c|}
\hline Catalyst & Solvent & $\begin{array}{l}\text { Time } \\
\text { (min) }\end{array}$ & $\begin{array}{c}\text { Yield } \\
(\%)\end{array}$ & Catalyst & Solvent & $\begin{array}{l}\text { Time } \\
(\mathrm{min})\end{array}$ & $\begin{array}{l}\text { Yield } \\
(\%)\end{array}$ \\
\hline & & & & & & & \\
\hline ZIF-8 & $\begin{array}{c}\text { Water } \\
\text { THF } \\
\text { Hexane } \\
\text { Ethanol }\end{array}$ & $\begin{array}{l}30 \\
30 \\
30 \\
30\end{array}$ & $\begin{array}{c}82 \\
65 \\
7 \\
82\end{array}$ & ZIF-8 & $\begin{array}{c}\text { DMF } \\
\text { Ethyl acetate } \\
\text { Toluene } \\
\text { DMSO }\end{array}$ & $\begin{array}{l}30 \\
30 \\
30 \\
30\end{array}$ & $\begin{array}{l}97 \\
49 \\
32 \\
92\end{array}$ \\
\hline
\end{tabular}

Reaction conditions: $25 \mathrm{mg}$ of ZIF-8, $5 \mathrm{~mL}$ of solvents, $4 \mathrm{mmol}$ of ethyl cyanoacetate, $3.5 \mathrm{mmol}$ of benzaldehyde, $80^{\circ} \mathrm{C}, 30 \mathrm{~min}$.

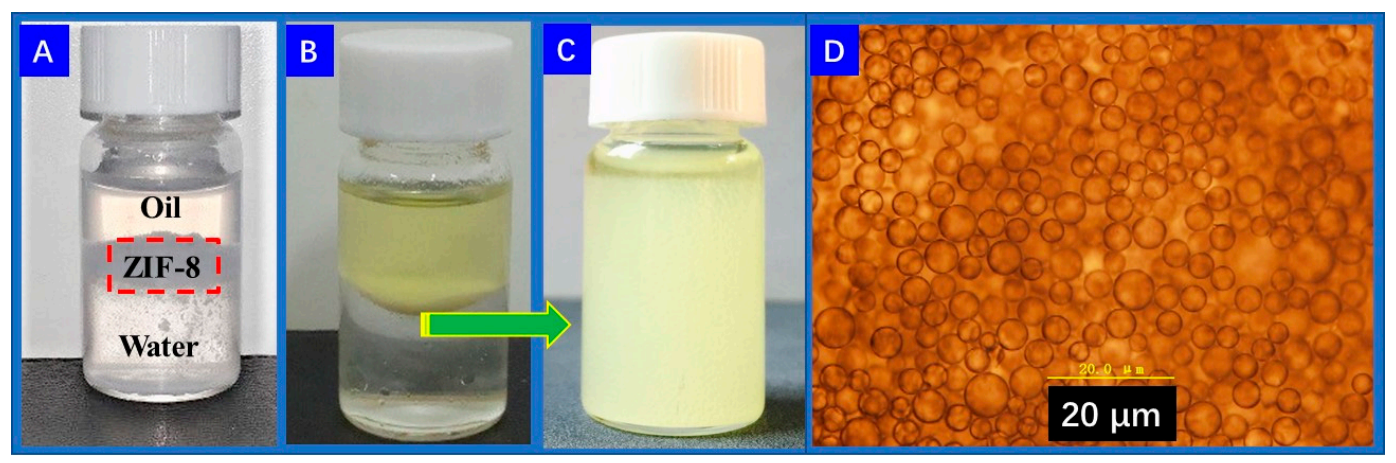

Figure 4. Photographs of the water/ZIF-8/ethyl acetate system (A) and water/ZIF-8/2-nitrobenzaldehyde and ethyl acetate system (B) without stirring. Appearance (C) and corresponding optical microscopy image (D) of Pickering emulsions stabilized by ZIF-8.

Having established that ZIF-8 is an efficient catalyst for the Knoevenagel condensation between benzaldehyde and ethyl cyanoacetate in an aqueous or biphasic system, we extended the study to various aromatic aldehydes with ethyl cyanoacetate or malononitrile. The results are listed in Table 2. To our delight, most of the aldehyde derivatives could be converted efficiently. Substitution and size effects were also observed. In our previous works, the effect of substitution on the reactivity of benzaldehyde catalyzed by UiO-66- $\mathrm{NH}_{2}$ was also observed for the Knoevenagel condensation [22,23]. The electron-donating substitutions lowered the catalytic efficiency, but the electron-withdrawing groups enhanced its catalytic efficiency. ZIF-8 was also active for the Knoevenagel reactions of larger aromatic aldehydes (1-naphthaldehyde and 9-anthraldehyde) with cyanoacetate or malononitrile, though the yields were somewhat decreased when the aldehyde substrates become bulkier (comparing Entries 1, 7 and 8 in Table 2). The decrease of yields may be an indicator of size effects. As shown by Entries 1, 7 and 8, the yield of 9-anthraldehyde reacting with ethyl cyanoacetate $(2 \mathrm{~h}, 23 \%)$ is much lower than that of 1-naphthaldehyde $(1 \mathrm{~h}, 45 \%)$, which is much lower than that of benzaldehyde $(1 \mathrm{~h}$, $>99 \%$ ). The activities vary in the size order of benzaldehyde $>$ 1-naphthaldehyde $>9$-anthraldehyde. This trend holds true for that of malononitrile. All results above reflect that the probability of the bulkier substrates forming transition-state complexes was significantly reduced due to the limited space in the porous catalyst. 
Table 2. ZIF-8-catalyzed Knoevenagel condensations of various substrates in the biphasic system.

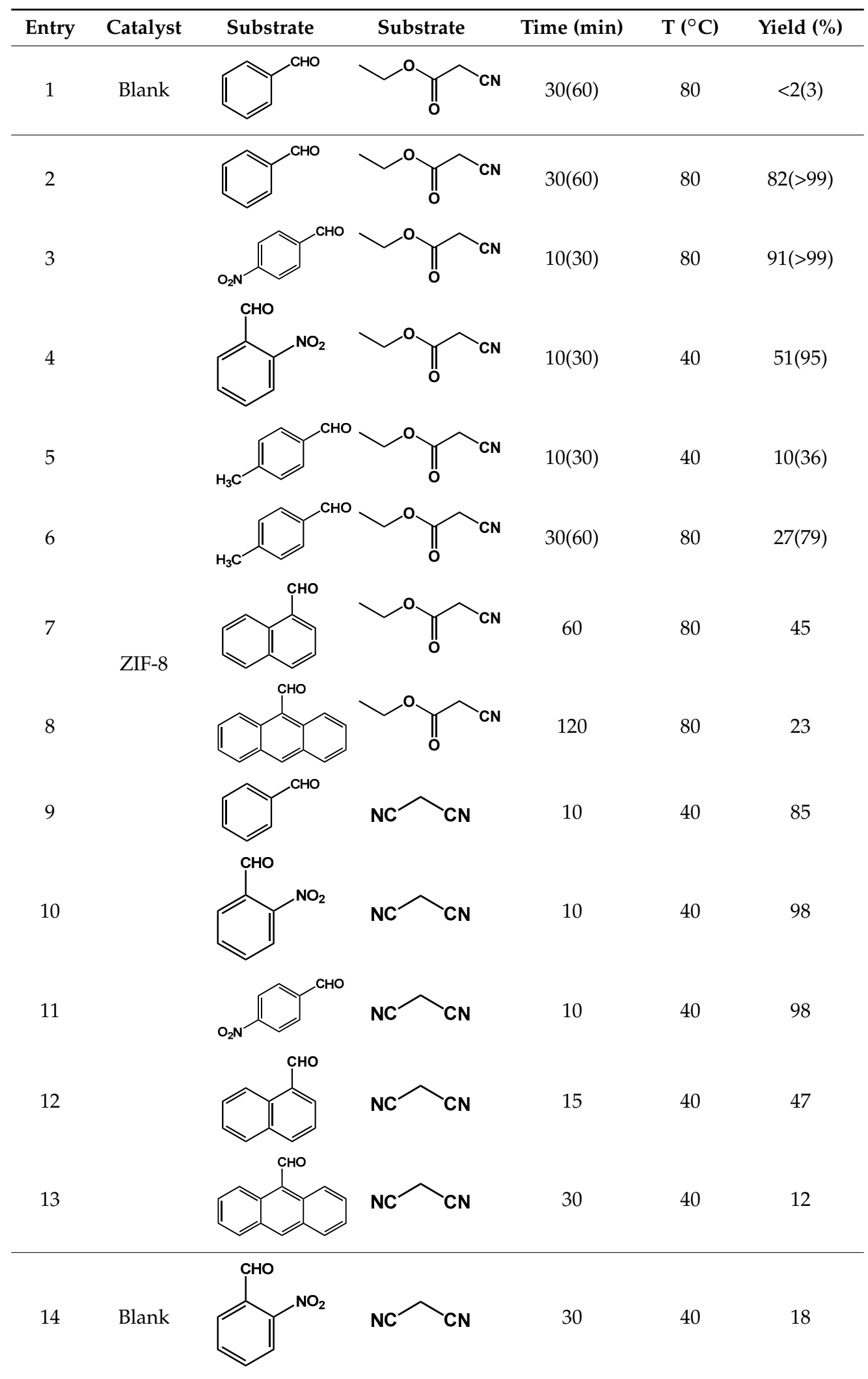

Reaction conditions: $3.5 \mathrm{mmol}$ aldehyde, $4 \mathrm{mmol}$ ethyl cyanoacetate or malononitrile, $25 \mathrm{mg}$ ZIF- 8 in $3 \mathrm{~mL}$ of $\mathrm{H}_{2} \mathrm{O}$ and $2 \mathrm{~mL}$ of ethyl acetate at 80 or $40^{\circ} \mathrm{C}$. 
The recyclability and reusability of solid catalysts are the most positive aspects compared to homogeneous catalysts for practical applications. Recovery and reuse of ZIF-8 was firstly studied using Knoevenagel condensation between benzaldehyde and ethyl cyanoacetate in a biphasic system at $80{ }^{\circ} \mathrm{C}$. After the completion of the reaction, the liquid layer was decanted, and the catalyst was reused for the next run under the same conditions. Furthermore, phase structures of recovered ZIF-8 were determined by powder X-ray diffraction. It was observed that the phase structure of ZIF-8 was completely destroyed after the reaction (Figure $5 b$ ). The following experiments were carried out to find out the possible destroyers. Previous results have proven that ZIF-8 is a water-stable MOF. Therefore, the stability of ZIF-8 in the aqueous solution of cyanoacetate and benzaldehyde was further tested, respectively. As shown in Figure 5c,d, the phase structure of ZIF-8 was maintained in the aqueous solution of cyanoacetate, but changed in that of benzaldehyde. The same results were observed even under mild reaction conditions by replacing ethyl cyanoacetate with malononitrile at $40^{\circ} \mathrm{C}$. The tested $\mathrm{pH}$ value of the aqueous solution of benzaldehyde was below five, indicating that the acid impurity was the possible destroyer of ZIF-8. Many reports indicated that the stability of ZIF-8 in aqueous medium is $\mathrm{pH}$ dependent. It is stable in a basic system, but instable in acidic medium [24,25]. Therefore, 2-nitro-benzaldehyde with high purity was selected as a new substrate. To our delight, as shown in Figure $6 c$, the XRD pattern of the recovered ZIF-8 is same as that of the fresh solid.

The recycle experiment was carried out using the Knoevenagel condensation between 2-nitrobenzaldehyde and malononitrile at $40^{\circ} \mathrm{C}$ in a biphasic system as a test reaction. Although some solid was lost during the centrifugation, as shown in Figure 7, the yields in the consecutive cycles were almost the same as those of the first cycle. XRD patterns of ZIF-8 before and afterreaction (Figure 8) revealed better integrity in the framework structure, but the TEM image (Figure 9c) demonstrated the presence of newly-formed mesoporous cages in the recovered solid. The retained or improved specific activity can probably be attributed to the more exposed active site and better mass transfer in the hierarchical ZIF-8. A hot filtration experiment was further performed to confirm the heterogeneous nature of the catalytic reaction (Figure 10). The solid catalyst was removed from hot solution by filtration $10 \mathrm{~min}$ after initiating the catalytic test run. The reaction of the filtrate was then monitored for another $50 \mathrm{~min}$. No significant further conversions were observed, indicating that most of the conversions were the result of the heterogeneous catalysis.

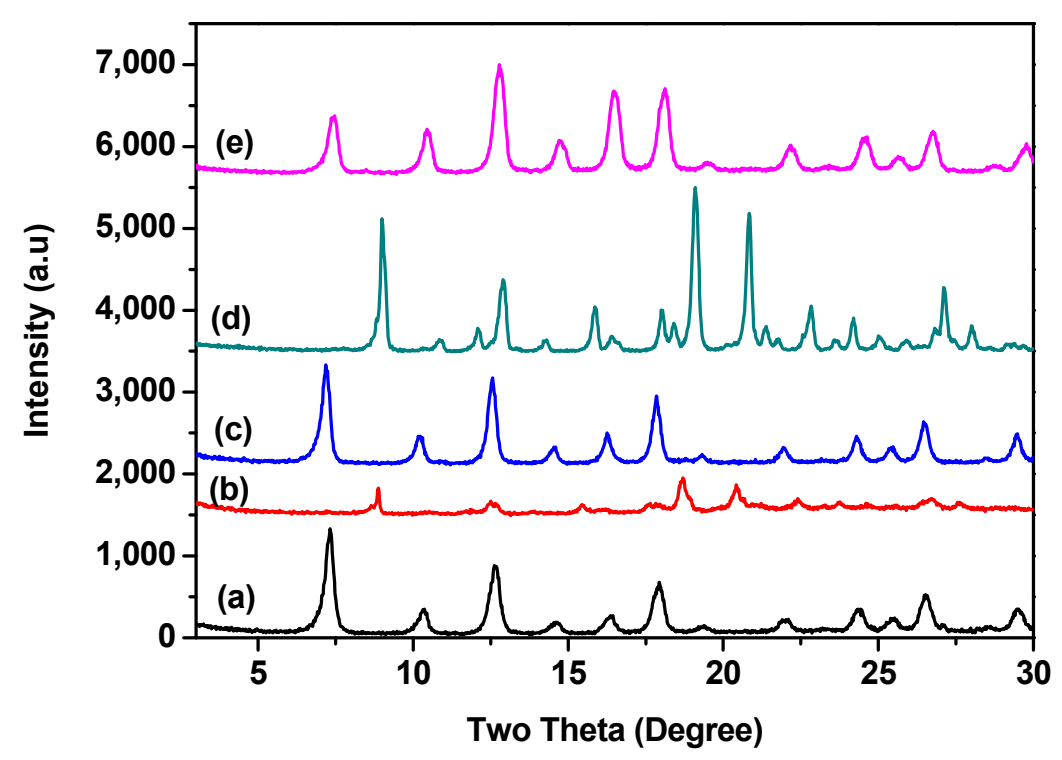

Figure 5. XRD patterns of fresh ZIF-8 (a), recovered ZIF-8 from the reaction between benzaldehyde and ethyl acetate in water at $80^{\circ} \mathrm{C}(\mathrm{b})$, ZIF- 8 treated with water at $80^{\circ} \mathrm{C}(\mathrm{c}), \mathrm{ZIF}-8$ treated with benzaldehyde in water at $80{ }^{\circ} \mathrm{C}(\mathbf{d}), \mathrm{ZIF}-8$ treated with ethyl acetate in water at $80{ }^{\circ} \mathrm{C}(\mathbf{e})$. 


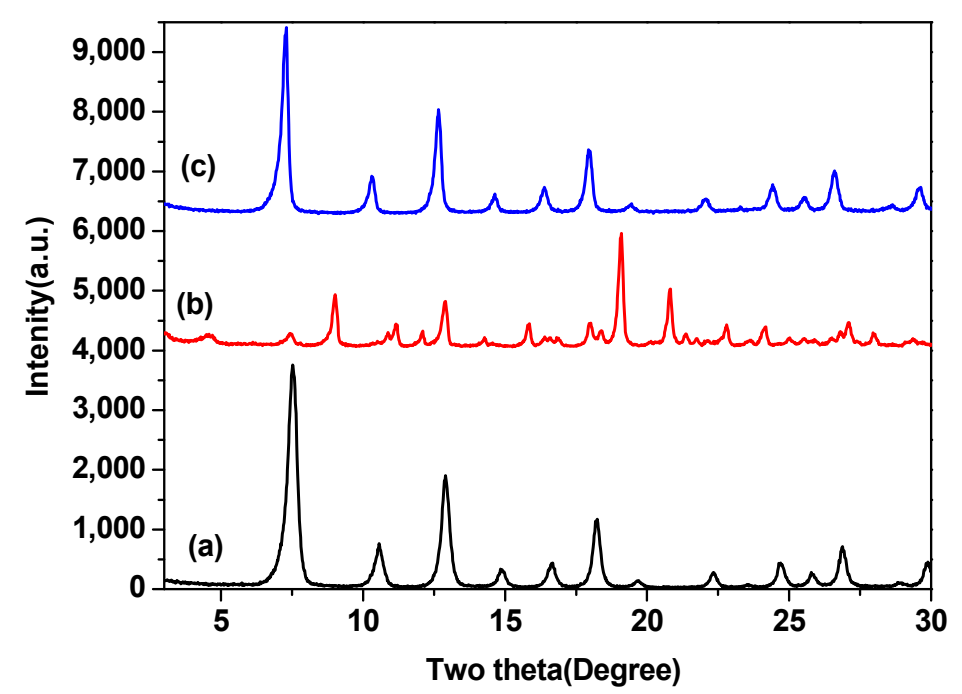

Figure 6. XRD patterns of fresh ZIF-8 (a), recovered ZIF-8 from the reaction between benzaldehyde and malononitrile in biphasic system at $40{ }^{\circ} \mathrm{C}(\mathbf{b})$ and recovered ZIF-8 from the reaction between 2-nitrobenzladehyde and malononitrile in biphasic system at $40^{\circ} \mathrm{C}$ (c).

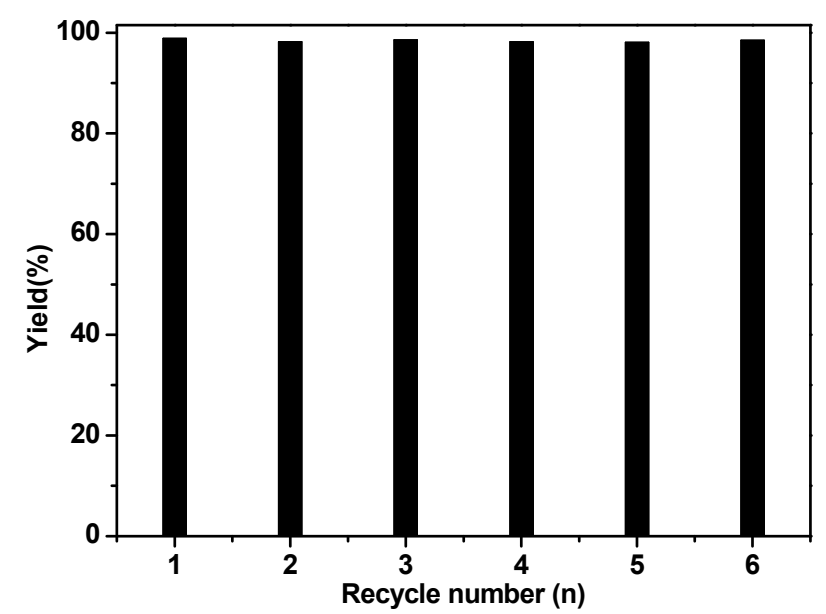

Figure 7. Recycle results of ZIF-8 in the Knoevenagel condensation between 2-nitrobenzaldehyde and malononitrile in biphasic system at $40{ }^{\circ} \mathrm{C}$.

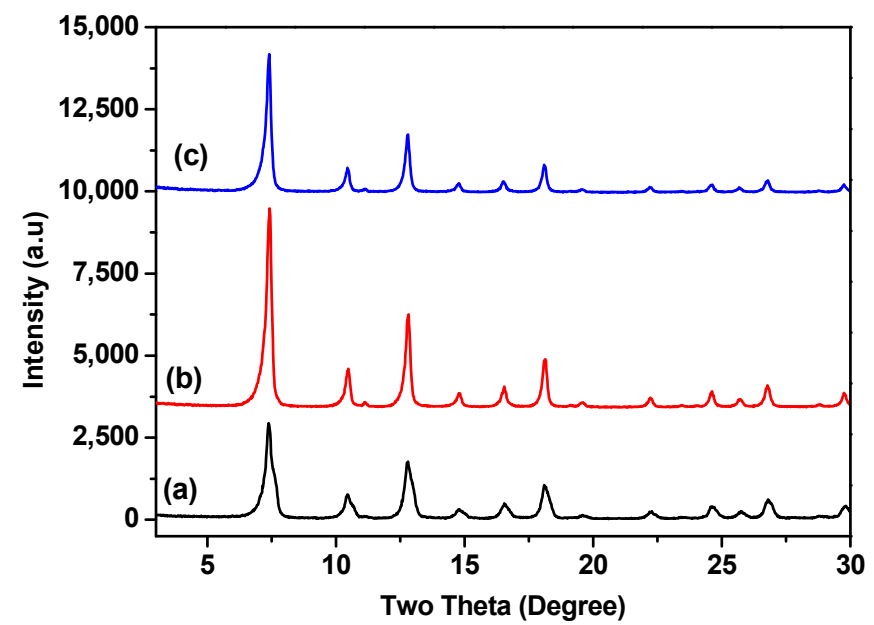

Figure 8. XRD patterns of fresh ZIF-8 (a), recovered ZIF-8 after three cycles (b) and after six cycles (c) from the reaction between 2-nitrobenzladehyde and malononitrile in biphasic system at $40{ }^{\circ} \mathrm{C}$. 

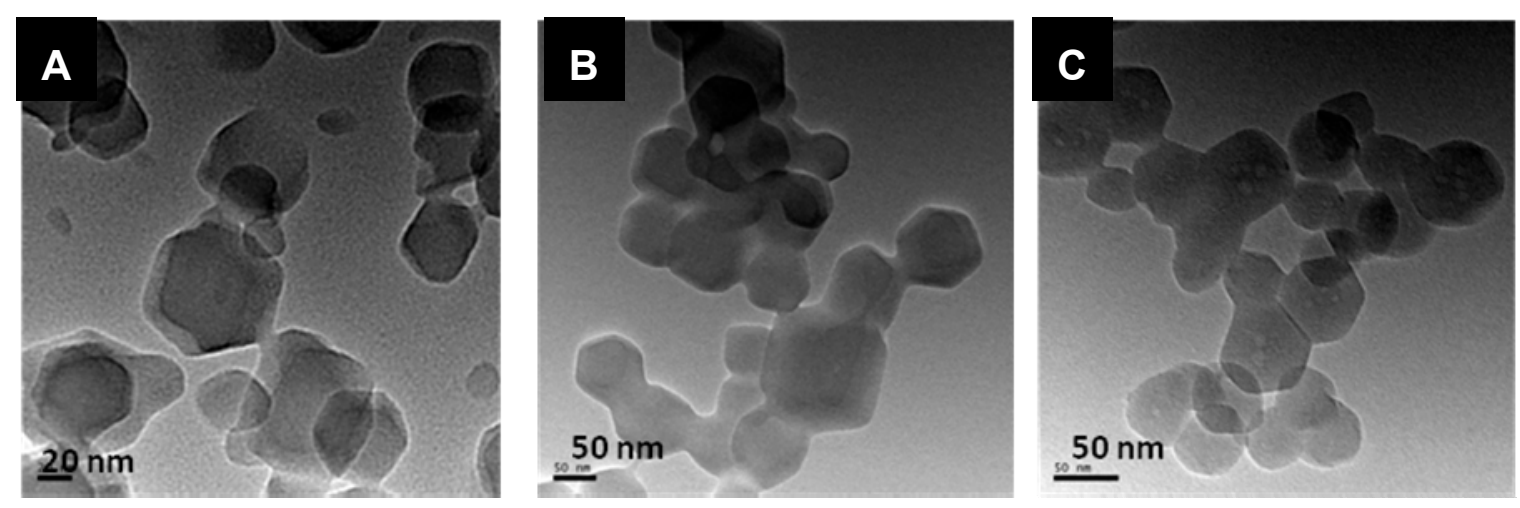

Figure 9. TEM images of fresh ZIF-8 (A), recovered after three cycles (B) and after six cycles (C) from the Knoevenagel condensation between 2-nitrobenzaldehyde and malononitrile in biphasic system at $40{ }^{\circ} \mathrm{C}$.

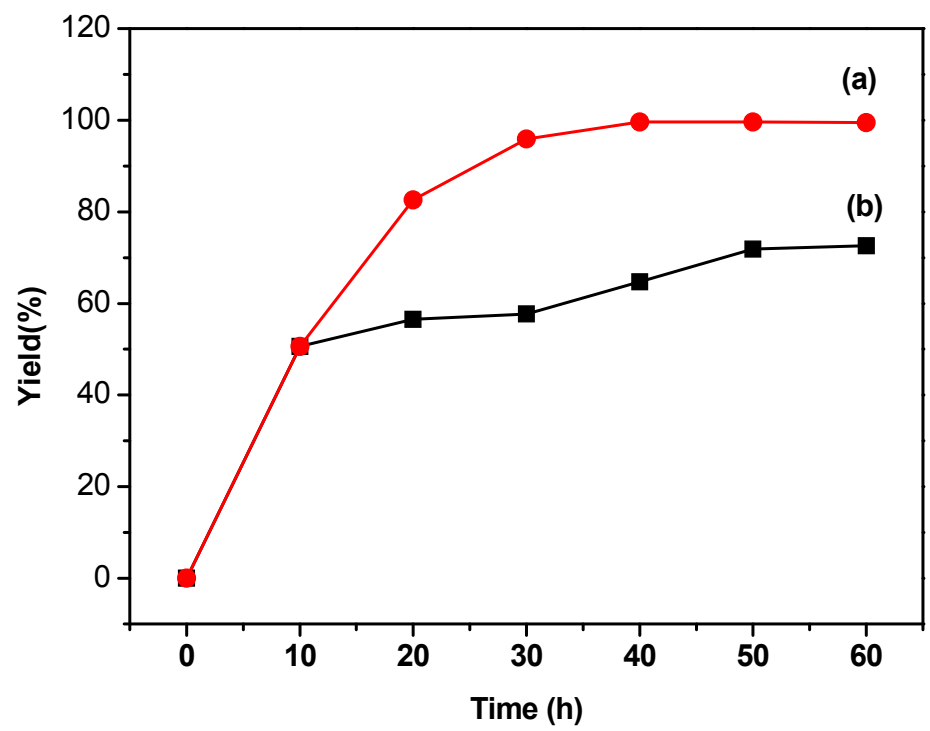

Figure 10. Hot filtration experiments of ZIF-8-catalyzed condensations between (a) 2-nitrobenzaldehyde and (b) ethyl acetate in a biphasic system.

\section{Experimental Section}

\subsection{Preparation of ZIF-8 Nanoparticles}

Synthesis of ZIF-8 nanoparticles: ZIF-8 nanoparticles were prepared according to the method reported with minor revision [21]. Firstly, 2-methylimidazole $(6.6 \mathrm{~g}, 80 \mathrm{mmol})$ and $\mathrm{Zn}\left(\mathrm{NO}_{3}\right)_{2} \cdot 6 \mathrm{H}_{2} \mathrm{O}(3 \mathrm{~g}$, $10 \mathrm{mmol}$ ) were dissolved in $100 \mathrm{~mL}$ of methanol, respectively. Then, the solution of $\mathrm{Zn}\left(\mathrm{NO}_{3}\right)_{2} \cdot 6 \mathrm{H}_{2} \mathrm{O}$ was quickly poured into the solution of 2-methylimidazole under stirring $(1000 \mathrm{rpm})$ at room temperature. After stirring for $1 \mathrm{~h}$, the precipitate was isolated by centrifugation at 10,000 rpm for $10 \mathrm{~min}$.The solid was washed three times with methanol and dried under a vacuum.

\subsection{Characterization}

The crystal structures of materials were analyzed by a Bruker D4 Endeavour Powder X-ray diffractometer $(\mathrm{Cu} \mathrm{K} \alpha, \lambda=0.15405 \mathrm{~nm}, 40 \mathrm{kV}$ and $40 \mathrm{~mA})$. The morphology and size of the ZIF-8 were determined by scanning electronic microscopy (SEM, HitachiS4800, Tokyo, Japan, $5.0 \mathrm{kV}$ ) and transmission electron microscopy (TEM, JEM-2100, Tokyo, Japan, 200 kV). The Pickering emulsions were measured on an Olympus BX51 microscope. 


\subsection{Catalytic Activity Measurements}

Typically, $25 \mathrm{mg}$ of ZIF-8, 4 mmol of ethyl cyanoacetate or malononitrile and $5 \mathrm{~mL}$ of solvents were added into a 10-mL glass reactor. After stirring for $10 \mathrm{~min}$ at the desired temperature under an inert atmosphere, $3.5 \mathrm{mmol}$ of benzaldehyde or derivatives were added to start the reaction. After the reaction, the catalyst was separated by centrifugation, successively rinsed with fresh ethyl acetate, ready for the next cycle. Yields of the reactions were estimated by gas chromatography (GC7900, Techcom, Shanghai, China) equipped with a flame ionization detector (FID) and an SE-30 capillary column $(30 \mathrm{~m} \times 0.25 \mathrm{~mm} \times 0.25 \mu \mathrm{m})$.

\section{Conclusions}

In summary, ZIF-8 was demonstrated to be a water stable and an interface-active catalyst for Knoevenagel condensation in a biphasic system. It efficiently promoted the Knoevenagel condensations of various aromatic aldehydes in biphasic systems as an interfacial catalyst. Pickering emulsions formed during the reaction due to its amphiphilic property, which was beneficial to mass transfer and enhanced its catalytic performance in a biphasic system. It exhibited good structural stability during the Knoevenagel condensation reactions and could be recovered and recycled as a heterogeneous catalyst for more than six times without obvious loss of activity in the condensation between 2-nitrobenzaldehyde and malononitrile at mild reaction conditions. The enhanced specific activity in the consecutive runs was ascribed to its newly-formed hierarchical structure observed by TEM images. Further development of catalytic reactions by using hierarchical ZIF-8 is currently under investigation. The findings mentioned above will open an avenue for using metal-organic frameworks as an interfacial solid catalyst.

Author Contributions: Y.Z., X.Z., and X.H. conceived and performed the experiments. Y.Z., R.B. and J.L. analyzed the data and wrote the paper.

Funding: This work was supported by the National Natural Science Foundation of China (21203017), the Open Fund of the State Key Laboratory of Catalysis, Dalian Institute of Chemical Physics, Chinese Academy of Sciences (N-11-3), the Program for Liaoning Excellent Talents in University (LNET-LJQ2014140) and the Fundamental Research Funds for the Central Universities (wd01201).

Conflicts of Interest: The authors declare no conflicts of interest.

\section{References}

1. Pera-Titus, M.; Leclercq, L.; Clacens, J.M.; Campo, F.; Nardello-Rataj, V. Pickering Interfacial Catalysis for Biphasic Systems: From Emulsion Design to Green Reactions. Angew. Chem. Int. Ed. 2015, 54, $2006-2021$. [CrossRef] [PubMed]

2. Zhou, W.J.; Fang, L.; Fan, Z.Y.; Albela, B.; Bonneviot, L.; De Campo, F.; Pera-Titus, M.; Clacens, J.M. Tunable Catalysts for Solvent-Free Biphasic Systems: Pickering Interfacial Catalysts over Amphiphilic Silica Nanoparticles. J. Am. Chem. Soc. 2014, 136, 4869-4872. [CrossRef] [PubMed]

3. Yang, H.Q.; Zhou, T.; Zhang, W.J. A Strategy for Separating and Recycling Solid Catalysts Based on the pH-Triggered Pickering-Emulsion Inversion. Angew. Chem. Int. Ed. 2013, 52, 7455-7459. [CrossRef] [PubMed]

4. Yang, T.Y.; Wei, L.J.; Jing, L.Y.; Liang, J.F.; Zhang, X.M.; Tang, M.; Monteiro, M.J.; Chen, Y.; Wang, Y.; Gu, S.; et al. Dumbbell-Shaped Bi-component Mesoporous Janus Solid Nanoparticles for Biphasic Interface Catalysis. Angew. Chem. Int. Ed. 2017, 56, 8459-8463. [CrossRef] [PubMed]

5. Rong, X.; Yang, H.Q.; Zhao, N. Rationally Turning the Interface Activity of Mesoporous Silicas for Preparing Pickering Foam and "Dry Water". Langmuir 2017, 33, 9025-9033. [CrossRef] [PubMed]

6. Chen, Z.W.; Zhou, L.; Bing, W.; Zhang, Z.J.; Li, Z.H.; Ren, J.S.; Qu, X.G. Light Controlled Reversible Inversion of Nanophosphor-Stabilized Pickering Emulsions for Biphasic Enantioselective Biocatalysis. J. Am. Chem. Soc. 2014, 136, 7498-7504. [CrossRef] [PubMed] 
7. Xue, W.; Yang, H.Q.; Du, Z.P. Synthesis of pH-Responsive Inorganic Janus Nanoparticles and Experimental Investigation of the Stability of Their Pickering Emulsions. Langmuir 2017, 33, 10283-10290. [CrossRef] [PubMed]

8. Kim, J.W.; Cho, J.; Cho, J.; Park, B.J.; Kim, Y.J.; Choi, K.H.; Kim, J.W. Synthesis of Monodisperse Bi-Compartmentalized Amphiphilic Janus Microparticles for Tailored Assembly at the Oil-Water Interface. Angew. Chem. Int. Ed. 2016, 55, 4509-4513. [CrossRef] [PubMed]

9. Zapata, P.A.; Faria, J.; Ruiz, M.P.; Jentoft, R.E.; Resasco, D.E. Hydrophobic Zeolites for Biofuel Upgrading Reactions at the Liquid-Liquid Interface in Water/Oil Emulsions. J. Am. Chem. Soc. 2012, 134, 8570-8578. [CrossRef] [PubMed]

10. Crossley, S.; Faria, J.; Shen, M.; Resasco, D.E. Solid Nanoparticles that Catalyze Biofuel Upgrade Reactions at the Water/Oil Interface. Science 2010, 327, 68-73. [CrossRef] [PubMed]

11. Hu, Y.H.; Wang, J.C.; Yang, S.; Li, Y.A.; Dong, Y.B. CuI@UiO-67-IM: A MOF-Based Bifunctional Composite TriphaseTransfer Catalyst for Sequential One-Pot Azide-Alkyne Cycloaddition in Water. Inorg. Chem. 2017, 56, 8341-8347. [CrossRef] [PubMed]

12. Huo, J.; Marcello, M.; Garai, A.; Bradshaw, D. MOF-Polymer Composite Microcapsules Derived from Pickering Emulsions. Adv. Mater. 2013, 25, 2717-2732. [CrossRef] [PubMed]

13. Farha, O.K.; Hupp, J.T. Rational Design, Synthesis, Purification, and Activation of Metal Organic Framework Materials. Acc. Chem. Res. 2010, 43, 1166-1175. [CrossRef] [PubMed]

14. Huo, J.; Aguilera-Sigalat, J.; El-Hankari, S.; Bradshaw, D. Magnetic MOF Microreactors for Recyclable Size-selective Biocatalysis. Chem. Sci. 2015, 6, 1938-1943. [CrossRef] [PubMed]

15. Wang, C.H.; Liu, X.L.; Demir, N.K.; Chen, J.P.; Li, K. Applications of water stable metal-organic frameworks. Chem. Soc. Rev. 2016, 45, 5107-5134. [CrossRef] [PubMed]

16. Burtch, N.C.; Jasuja, H.; Walton, K.S. Water stability and adsorption in metal-organic frameworks. Chem. Rev. 2014, 114, 10575-10612. [CrossRef] [PubMed]

17. Schejn, A.; Mazet, T.; Falk, V.; Balan, L.; Aranda, L.; Medjahdi, G.; Schneider, R. Fe $\mathrm{O}_{4} @$ ZIF-8: Magnetically recoverable catalysts by loading $\mathrm{Fe}_{3} \mathrm{O}_{4}$ nanoparticles inside a zinc imidazolate framework. Dalton Trans. 2015, 44, 10136-10140. [CrossRef] [PubMed]

18. Chebbat, N.; Commenge, J.M.; Medjandi, G.; Schneider, R. ZIF-8 nanoparticles as an efficient and reusable catalyst for the Knoevenagel synthesis of cyanoacrylates and 3-cyanocoumarins. Tetrahedron Lett. 2016, 57, 5885-5888.

19. Guan, Y.B.; Shi, J.J.; Xia, M.; Zhang, J.; Pang, Z.F.; Marchetti, A.; Wang, X.H.; Cai, J.S.; Kong, X.Q. Monodispersed ZIF-8 particles with enhanced performance for $\mathrm{CO}_{2}$ adsorption and heterogeneous catalysis. Appl Surf. Sci. 2017, 423, 349-535. [CrossRef]

20. Nguyen, L.L.; Ky, L.E.; Phan, N.T.S. A Zeolite Imidazolate Framework ZIF-8 Catalyst for Friedel-Crafts Acylation. Chin. J. Catal. 2012, 33, 688-696. [CrossRef]

21. Tsai, C.W.; Langner, E.H.G. The effect of synthesis temperature on the particle size of nano-ZIF-8. Microporous Mesoporous Mater. 2016, 221, 8-13. [CrossRef]

22. Zhang, Y.M.; Zhang, J.; Tian, M.M.; Quan, C.S.; Fan, S.D. Fabrication of Amino-functionalized $\mathrm{Fe}_{3} \mathrm{O}_{4} @ \mathrm{Cu}_{3}(\mathrm{BTC})_{2}$ for Heterogeneous Knoevenagel Condensation. Chin. J. Catal. 2016, 37, 420-427. [CrossRef]

23. Zhang, Y.M.; Dai, T.L.; Zhang, J.; Chu, G.; Quan, C.S. $\mathrm{Fe}_{3} \mathrm{O}_{4} @ U$ UiO-66- $\mathrm{NH}_{2}$ Core-Shell Nanohybrid as Stable Heterogeneous Catalyst for Knoevenagel Condensation. Chin. J. Catal. 2016, 37, 2106-2113. [CrossRef]

24. Li, P.Z.; Aranishi, K.; Xu, Q. ZIF-8 immobilized nickel nanoparticles: Highly effective catalysts for hydrogen generation from hydrolysis of ammonia borane. Chem. Commun. 2012, 48, 3173-3175. [CrossRef] [PubMed]

25. Sun, C.Y.; Sun, C.Y.; Qin, C.; Wang, X.L.; Yang, G.S.; Shao, K.Z.; Lan, Y.Q.; Su, Z.M.; Huang, P.; Wang, C.G.; et al. Zeolitic imidazolate framework- 8 as efficient $\mathrm{pH}$-sensitive drug delivery vehicle. Dalton Trans. 2012, 41, 6906-6909. [CrossRef] [PubMed]

(C) 2018 by the authors. Licensee MDPI, Basel, Switzerland. This article is an open access article distributed under the terms and conditions of the Creative Commons Attribution (CC BY) license (http:/ / creativecommons.org/licenses/by/4.0/). 